\title{
Sobre el concepto de ley permisiva y su función argumentativa en el § 2 de la Rechtslehre
}

\author{
Fiorella Tomassini \\ Pós-graduanda em Filosofia na Universidade \\ de Buenos Aires, Argentina
}

Resumo: Este trabajo se propone analizar por qué Kant señala en el § 2 de la Rechtslehre que el postulado jurídico de la razón práctica puede denominarse una ley permisiva, considerando que luego de esa mención el autor no ofrece ninguna definición ulterior ni desarrollo teórico de ese concepto. El modo en que se interpreta la función argumentativa del concepto jurídico de lex permissiva en el desarrollo de la doctrina de la propiedad, que Kant presenta en la sección "El derecho privado" del texto que nos ocupa, tiene consecuencias fundamentales en la comprensión de la relación entre la propiedad y el Estado en el pensamiento político de Kant.

Palavras-chave: propiedad; ley permisiva; derecho privado; Kant.
Ahstract: This paper analyzes why Kant states in $\S 2$ of the Rechtslehre that the postulate of practical reason can be called a permissive law, considering that after that the author offers no further definition nor theoretical development of that concept. The way the argumentative function of the concept of lex permissiva is interpreted in the development of the doctrine of property, which Kant presents in the section "Private right" of the mentioned text, has fundamental consequences for the understanding of the relationship between property and State in Kant's political thinking.

Keywords: property; permissive law; private right; Kant.

Con su célebre trabajo "Das Erlaubnisgesetz oder: Vernunft und Geschichte in Kants Rechtslebre", Reinhard Brandt fue el primer autor en notar la importancia del concepto de ley permisiva en la Rechtslebre, pues hasta ese momento, según relata, ni ese concepto ni el postulado jurídico de la razón práctica habían sido objeto de estudio de la 
literatura especializada. ${ }^{1}$ En líneas generales, Brandt propone que la función de la lex permissiva en la extensa primera sección de la Rechtslebre, "El derecho privado", debe entenderse en continuidad con el uso de ese concepto jurídico en Zum ewigen Frieden - como un modo de otorgarle un carácter jurídico a la adquisición provisoria hasta que exista la posibilidad de un cambio o reforma-. Frente al hecho de que los Estados existentes no se adecúan al estado de derecho, la ley permisiva permitiría aplicar "normas naturales o jurídico-racionales" a un estado de cosas injusto hasta que sea posible una reforma paulatina. ${ }^{2}$ En su reciente libro Kant's Doctrine of Right. A commentary, Sharon Byrd y Joachim Hruschka han discutido la clásica lectura de Brandt sobre esta cuestión. ${ }^{3}$ Estos autores sostienen que el concepto de ley permisiva adquiere en la Rechtslebre un sentido novedoso con respecto a su mención en Zum ewigen Frieden, y que éste debe entenderse en su versión ulterior como una "norma que confiere poderes" que otorga "una facultad moral o capacidad para ser propietario de objetos físicos". ${ }^{4}$ Ambas líneas interpretativas convergen, no obstante, en la aseveración de que en la teoría jurídica kantiana la propiedad es una institución previa al establecimiento del Estado, autorizada por la razón práctica

1. BRANDT, R. Das Erlaubnisgesetz oder: Vernunft und Geschichte in Kants Rechtslehre. In: Rechtspbilosopbie der Aufklärung: Symposium Wolfenbüttel. Berlin: Walter de Gruyter, 1982, p. 235.

2. Por otra parte, Kant desarrollaría esta noción de ley permisiva - sostiene Brandt - de cara a acontecimientos históricos (la Revolución Francesa y la Reforma Dinástica en Prusia y Alemania). La postura política de Kant frente a estos acontecimientos consistiría en abogar por la aplicación de normas jurídicas mediante una "reforma paulatina", y no mediante una revolución del pueblo (como el caso francés) ni del príncipe (como el caso prusiano). Idem, p. 250 e ss.

3. BYRD, S./ HRUSCHKA, J. Kant's Doctrine of Rigbt. A Commentary. Cambridge: Cambridge University Press, 2010. Cf. BYRD, S. Intelligible possession of objects of choice. In: DENIS, L. (comp.). Kant's Metapbysical of Morals. Cambridge: Cambridge University Press, 2010; HRUSCHKA, J. The Permissive Law of Practical Reason in Kant's 'Metaphysics of Morals'. Law and Pbilosopby, v. 23, n. 1, 2004. pp. 45-72. Las lecturas de Kersting y Tierney sobre la ley permisiva siguen en buena medida la tesis de Brandt (KERSTING, W. Wolbgeordnete Freibeit. Frankfurt am Main: Suhrkamp, 1993, pp. 248-249; TIERNEY, B. Kant on Property: The Problem of Permissive Law. Journal of the History of Ideas, v. 62, n. 2, 2001. pp. 301-312).

4. BYRD, S./ HRUSCHKA, J. Kant's Doctrine of Right, p. 106. 
a través de una ley permisiva (cuyo poder para establecer derechos relativos a la adquisición privada tiene un carácter provisorio en la lectura de Brandt, y definitivo, en la lectura de Byrd y Hruschka). Con ello, sendas lecturas coinciden en otorgarle una primacía al derecho privado frente al derecho público, entendiendo que la función del Estado kantiano se reduce a proteger y regular la propiedad.

Frente a una lectura de la ley permisiva en la Rechtslebre a partir de sus usos en textos precedentes — como la propuesta por Brandt-, considero importante remarcar que Kant utiliza este concepto jurídico en un contexto argumentativo diferente al de su mención en el escrito político de 1795. En efecto, el parágrafo $§ 2$ de la sección "El derecho privado", en el cual el postulado jurídico de la razón práctica es denominado "ley permisiva", se inserta en la primera de las dos secciones ("el modo de tener algo exterior como suyo" §§ 1-9 y "el modo de adquirir algo exterior" $§ \S 10-17)$ que conforman la doctrina kantiana de la propiedad. Se trata, ahora, del plano teórico y normativo en el que se desarrolla el análisis de la posibilidad de relaciones jurídicas de hombres libres en relación a objetos exteriores ("lo mío y tuyo externo"), y no, como en Zum ewigen Frieden, del tópico de la relación entre su teoría normativa del derecho y la práctica política en los Estados existentes. Por ello, en este trabajo asumo como punto de partida que el concepto de ley permisiva en la Rechtslebre debe ser comprendido atendiendo tanto la metodología específica y el carácter sistemático que presupone la elaboración de una filosofía del derecho y del Estado, que es el contexto argumentativo en el cual Kant utiliza ese concepto jurídico

Por otra parte, toda interpretación de la doctrina kantiana de la propiedad según la cual la adquisición privada se funda en principios racionales a priori del derecho privado, independientemente de la instancia de la soberanía de la voluntad general, — tesis a la que adscribirían tanto Brandt como Byrd y Hruschka-, es, a mi modo de ver, insatisfactoria por dos razones centrales. En primer lugar, Kant niega que el así llamado "derecho a la propiedad" tenga un carácter natural y defiende que es siempre un derecho adquirido, y como tal, requiere un acto jurídico para su validez, en oposición al único derecho innato "que corresponde a cada uno por naturaleza". 5 Según la definición

5. RL, p. 237. Cito las obras de Kant según la paginación canónica de la AkademieAusgabe, Kant's gesammelte Schriften. La referencia se indica por medio de la sigla 
mencionada, la validez de los derechos adquiridos se vincula necesariamente con la idea de un estado jurídico. En segundo lugar, cuando se examina atentamente la metodología en la justificación de los deberes que supone la teoría jurídica kantiana-que exige la universalidad, necesidad y reciprocidad en sus principios-, el postulado jurídico de la razón práctica, formulado como una ley permisiva, se descubre como un principio normativo insuficiente para establecer derechos en relación a "lo mío y tuyo exterior". En efecto, como veremos más adelante, este principio jurídico no podría garantizar la igualdad y reciprocidad de la obligación prescripta por el derecho innato.

A partir de estas consideraciones, en este trabajo sostendré que el concepto de ley permisiva del $\S 2$ de la Rechtslebre debe ser estudiado teniendo en cuenta la especificidad y el rol argumentativo que adquiere la teoría jurídica kantiana en esa obra, y especialmente, dentro del contexto sistemático de la sección "El derecho privado", que es la sección en la cual Kant se ocupa de los derechos adquiridos como derechos subjetivos ${ }^{6}$, esto es, como facultades o capacidades morales que tienen las personas de obligar a los demás, ${ }^{7}$ pero que a su vez requieren necesariamente de un acto jurídico para su validez. En ese marco,

correspondiente y, a continuación, se señala el número de página. Para citar Die Metapbysik der Sitten, utilizo la siguiente versión castellana: La metafísica de las costumbres, prólogo, traducción y notas: Adela Cortina Orts. Barcelona: Ediciones Altaya, 1993. Indicaré los casos en que la traducción haya sido modificada.

6. Kant establece en la "Introducción a la Doctrina del derecho" una "división general de los derechos" (RL, p. 236): los derechos, considerados como preceptos sistemáticos (derechos objetivos), se dividen en derecho positivo y derecho natural, y los derechos, considerados como facultades de coaccionar (derechos subjetivos), se dividen en derecho innato y derechos adquiridos. Estos últimos pueden denominarse asimismo "lo mío y tuyo interno" [innere Mein und Dein] y "lo mío y tuyo externo" [äußere Mein und Dein], respectivamente. De este modo, la sección titulada "el Derecho privado sobre lo mío y tuyo externo en general" ["das Privatrecht vom üußeren Mein und Dein überhaupt"], como su nombre lo indica, se restringe a los derechos adquiridos como parte de los derechos subjetivos y no al ámbito del derecho natural (para Kant, la idea de derecho natural engloba los principios jurídicos a priori tanto aquellos establecidos en el derecho privado como en el derecho público ( $R L$, p. 242)).

7. RL, p. 237. 
intentaré defender la tesis de que el concepto de ley permisiva denota, en el parágrafo que nos ocupa, una validez meramente subjetiva y que, por lo tanto, no es suficiente para imponer una obligación para terceros de manera legítima. La potestad conferida por la ley permisiva constituiría, según mi hipótesis interpretativa, un título racional o fundamento para demandar la institución de un Estado legislado por la voluntad general con el fin de que sea posible gozar legítimamente de derechos relativos a la propiedad.

Antes de analizar con detalle la denominación del postulado jurídico de la razón práctica como una ley permisiva (III), comenzaré por analizar brevemente la función sistemática de ese postulado en la doctrina de la posesión ("el modo de tener algo exterior como suyo", $\S \S 1-9)($ II).

\section{II}

La primera sección de "El derecho privado" ("el modo de tener algo como suyo" $\S \S 1-9)$ tiene como objetivo central demostrar la necesidad del concepto de posesión jurídica como condición necesaria para desarrollar una doctrina de "lo mío y tuyo exterior". Esto significará mostrar, a su vez, "cómo es posible una proposición jurídica sintética a priori" ${ }^{8}$ Kant tiene que establecer un principio jurídico que autorice en general la posesión de objetos, porque ni del principio universal del derecho ni del derecho innato puede derivarse algún derecho o permiso al uso de objetos. Con ese fin, Kant comienza el $\$ 2$ con la formulación del postulado ${ }^{9}$ jurídico de la razón práctica.

8. RL, p. 249.

9. El término "postulado" no es empleado por Kant de manera unívoca. Guyer señala que a la luz de $\operatorname{KrV}$ (A 633-4 B 661-2) un postulado es una proposición teórica que afirma la existencia de un objeto o de un estado de cosas que son condición de posibilidad de la fuerza obligante de un deber moral (GUYER, P. Kant's Deduction of the Principle of Rights. In: TIMMONS, M. (ed.). Kant's Metaphysics of Morals Interpretative Essays. Oxford: Oxford University Press, 2002, p. 37). Basándose en la segunda Crítica, Ludwig sugiere que los postulados deben entenderse del siguiente modo: "objetos de postulados de la razón práctica son las condiciones necesarias de realización de las exigencias que se derivan del imperativo categórico, tanto la inmortalidad del alma, dios, como la ley jurídica y lo mío y tuyo en relación con la posesión 
Uno de los obstáculos que se presenta al reconstruir la argumentación consiste en que el postulado es formulado de tres diversos modos: a comienzos del $§ 2$ como una aserción — "es posible tener como mío cualquier objeto exterior de mi arbitrio"10_, luego como una ley permisiva - "este postulado puede llamarse una ley permisiva de la razón práctica"11 - y, por último, en el $§ 6$, como una prescripción - "es deber jurídico actuar con respecto a otros de tal modo, que lo exterior útil pueda llegar a ser también para cualquiera suyo" $^{\prime 12}$ - . Como veremos a continuación, las formulaciones del postulado no son equivalentes entre sí sino que cada una de ellas adquiere un sentido específico cuando se tiene en cuenta el contexto argumentativo. Comencemos por analizar la primera formulación:

Es posible tener como mío cualquier objeto exterior de mi arbitrio; es decir, es contraria al derecho una máxima según la cual, si se convirtiera en ley, un objeto del arbitrio tendría que ser en sí (objetivamente) un objeto sin dueño (res nullius). ${ }^{13}$

Luego de introducir el postulado, Kant procede a su demostración por reducción al absurdo. El punto central de la argumentación es mostrar que lo contrario a lo que afirma el postulado, esto es, una prohibición de "tener como mío cualquier objeto exterior de mi arbitrio", supondría una "contradicción de la libertad externa consigo misma". ${ }^{14}$ Examinemos por qué.

Kant restringe el ámbito del derecho a la interacción práctica de los hombres sin atender a los fines de sus acciones. ${ }^{15}$ Este requisito metodológico debe ser respetado por todos los principios jurídicos de la Rechtslebre. Como explica Kersting, en el caso de un principio jurídico vinculado con el uso de objetos exteriores al arbitrio, esto significa que "no es jurídicamente relevante qué fin quiere perseguir el arbitrio,

inteligible". LUDWIG, B. Kants Recbtslebre. Hamburg: Felix Meiner, 2005, p. 111.

10. RL, p. 246

11. RL, p. 247.

12. RL, p. 252.

13. RL, p. 246.

14. RL, p. 246.

15. RL, p. 320 . 
ni tampoco qué características tiene el objeto del arbitrio, lo único relevante desde la perspectiva de la razón jurídica es que algo sea un objeto del arbitrio". ${ }^{16} \mathrm{Si}$ lo único relevante desde la perspectiva del derecho es que algo sea o no un objeto del arbitrio (y no cual sea el fin del uso de ese objeto o sus características), un principio jurídico solo podrá determinar, o bien la posibilidad general de la posesión de objetos ("algo puede ser objeto del arbitrio"), o bien la prohibición de la posesión de objetos ("nada puede ser objeto del arbitrio"). Así, desde la perspectiva de la razón práctica jurídica, quedan dos posibilidades excluyentes: la posibilidad general de la posesión o su prohibición absoluta. Kant sostiene que la segunda de esas opciones es absurda y, por lo tanto, que es correcto postular "es posible tener como mío cualquier objeto de mi arbitrio ${ }^{17}{ }^{17}$ En palabras de Kant:

Pero, siendo así que la razón pura práctica no adopta sino leyes formales del uso del arbitrio, y por tanto, abstrae de la materia del arbitrio, es decir, de la restante constitución del objeto, con tal de que sea un objeto del arbitrio, no puede ésta contener ninguna prohibición absoluta de usar tal objeto, porque esto sería una contradicción de la libertad consigo misma. ${ }^{18}$

Restaría explicar por qué una prohibición absoluta de la posesión de objetos externos al arbitrio supondría una contradicción de la libertad consigo misma. Kant argumenta del siguiente modo. Primero señala que un "objeto de mi arbitrio" es aquello que "está en mi poder físicamente" y algo que tengo "capacidad física de usar discrecionalmente" ${ }^{19}$ Ahora bien, si ese objeto de mi arbitrio, el cual tengo en mi poder "físicamente", no pudiera estar en mi "poder jurídicamente", esto es "si usarlo no fuera compatible (fuera injusto) con la libertad de cualquier otro según una ley universal: entonces la libertad se privaría a sí misma de usar su arbitrio con relación a un objeto de él, al imposibilitar el uso de objetos utilizables". ${ }^{20}$ Es decir, si una ley práctico-jurídica enunciara una prohibición según la cual la libertad deba privarse a sí misma de usar su arbitrio en relación a los

16. KERSTING, W. Wolhgeordnete Freibeit, p. 244.

17. RL, p. 245.

18. RL, p. 246.

19. $R L$, p. 246.

20. RL, p. 246. 
objetos exteriores, los cuales son de becho objetos de mi arbitrio —en el sentido de que "están en mi poder físicamente" y tengo "capacidad de usar discrecionalmente" - , convirtiendo esos objetos en res nullius, ${ }^{21}$ la libertad caería en contradicción consigo misma. Por lo tanto, concluye Kant, "es una presuposición a priori de la razón práctica considerar y tratar cualquier objeto de mi arbitrio como mío y tuyo objetivamente posibles". 22

La doctrina de la posesión jurídica, como anteriormente mencioné, está centrada en mostrar que "el modo de tener algo como suyo" es la posesión jurídica y no la posesión empírica. La deducción del concepto de posesión jurídica no se establece sino hasta $R L \S 6$, donde la posibilidad de ese concepto, y con ello la posibilidad de una proposición jurídica sintética a priori, queda fundada de modo definitivo en el deber jurídico de actuar en conformidad con el postulado jurídico de la razón práctica. Dice Kant:

La posibilidad de una posesión semejante, y con ello la deducción del concepto de una posesión no empírica, se basa en el postulado jurídico de la razón práctica: "es deber jurídico actuar con respecto a otros de tal modo, que lo exterior (útil) pueda llegar a ser también para cualquiera suyo". ${ }^{23}$

En esta segunda versión, el postulado es presentado no ya como una aserción que enuncia una posibilidad, como la primera definición en $\S 2$, sino bajo la forma de un imperativo. Esta reformulación del postulado como un deber permite a Kant enfatizar que el punto central de la deducción del concepto de posesión jurídica consiste en que "si es necesario actuar según aquel principio jurídico, entonces también tiene que ser posible la condición inteligible (de una posesión meramente jurídica)". El deber jurídico de actuar según "principios de lo mío y tuyo exterior", de modo que los objetos puedan ser "míos o tuyos", contiene analíticamente en su enunciación el concepto jurídico a priori necesario para que algo exterior pueda ser objeto de posesión, a saber, el concepto de posesión inteligible. En caso contrario, esto es, si no se supone una posesión no empírica se anularía la posibilidad de adquirir objetos, y, en consecuencia, no podría cumplirse una

21. RL, p. 246.

22. RL, p. 246.

23. RL, p. 252. 
exigencia de la razón práctica. Sería absurda la existencia de deberes jurídicos si las condiciones para cumplirlos fueran racionalmente imposibles. De acuerdo con Kant, el concepto de posesión jurídica es una consecuencia inmediata del deber jurídico introducido en esta segunda formulación del postulado, y con ello queda debidamente fundamentado.

En la próxima sección, nos tocará entonces analizar con detalle la tercera formulación del postulado de la razón práctica: la denominación de este principio jurídico como una "ley permisiva de la razón práctica". ${ }^{24}$

\section{III}

En el apartado titulado "Conceptos preliminares de la Metafísica de las costumbres" de la "Introducción a la Metafísica de las costumbres", Kant se pregunta si conforme a la distinción entre acciones prohibidas, obligatorias y permitidas, debe incluirse entre las leyes morales, junto a la noción de ley prohibitiva (lex probibitiva) y de ley preceptiva (lex praeceptiva, lex mandati), la idea de una ley permisiva (lex permissiva). Dice Kant:

Una acción que no está ordenada ni prohibida está simplemente permitida, porque con respecto a ella no hay ley alguna que limite la libertad (permisión) ni, por tanto, deber alguno. Una acción semejante se llama moralmente-indiferente (indifferens, adiaphoron, res merae facultatis). Podemos preguntarnos si hay acciones semejantes y si, en el caso de que las haya, es necesaria todavía una ley permisiva (lex permissiva) para que a alguien le esté permitido hacer u omitir a su gusto, además de la ley preceptiva (lex praeceptiva, lex mandati) y de la ley prohibitiva (lex probibitiva, lex vetiti). Si así fuera, el permiso no se referiría en todo caso a una acción moralmente indiferente (adiapboron); porque para una acción semejante no se precisaría ley especial alguna, si la consideramos desde la perspectiva de las leyes morales. ${ }^{25}$

Kant introduce esta discusión acerca de las leyes permisivas a partir de la clasificación de las acciones, y en particular, en relación con las acciones "moralmente indiferentes". Una ley permisiva solo sería necesaria si entre aquellas acciones meramente permitidas, esto

24. RL, p. 247.

25. RL, p. 223. 
es, ni prohibidas ni obligatorias, hubiera algunas que no fueran adiaphora o indifferens, y con ello, requirieran de un permiso. En este pasaje de la "Introducción" Kant solo deja asentado, entonces, en qué caso debería incluirse el concepto de lex permissiva en la clasificación de las leyes morales y no se pronuncia acerca de si se da o no el caso. De todos modos, sabemos que la respuesta es afirmativa, puesto que esta noción aparecerá en ambas doctrinas, tanto en la Rechtslebre, en los parágrafos $\S 2$ y $§ 16$, como en la Tugendlebre. ${ }^{26}$

En el $§ 2$ de la sección "El derecho privado", Kant afirma que el postulado de la razón práctica, que tenía la función de probar la posibilidad moral —en contraposición a una posibilidad meramente física que no necesita demostración alguna- de hacer uso de los objetos según el concepto de posesión jurídica, puede llamarse una ley permisiva de la razón que

nos confiere la potestad ${ }^{27}$ que no podríamos extraer de los meros conceptos del derecho en general; a saber, imponer a todos los demás una obligación que no tendrían de no ser así: la de abstenerse

26. TL, p. 426.

27. Traduzco "potestad" por Befugnis porque es el término castellano que mejor refleja la idea de "facultad o capacidad moral" de hacer algo que está en juego en el término alemán que utiliza Kant. La palabra "competencia", elegida por Cortina Orts, remite más a la idoneidad para hacer algo y no recoge el matiz moral que tiene la palabra alemana. El término "authorization", utilizado en la mayoría de las traducciones inglesas, incluida la Metapbysics of Morals de Mary Gregor, no refleja su aspecto subjetivo. La relevancia de la traducción del término Befugnis no es menor: que una persona tenga "la capacidad o facultad moral" para hacer algo no supone necesariamente que tenga una autorización, en este caso jurídica, para hacerlo; sino más bien un título o fundamento racional mediante el cual reclamarla. No hay que olvidar que este término aparece aquí en el contexto del "derecho privado" donde Kant argumenta acerca de los derechos adquiridos como facultades o capacidades morales. La noción de "derecho subjetivo" surge precisamente en la historia del derecho natural cuando se vinculó el término ius [Recbt], entendido como facultas o qualitas moralis [Vermögen], con la noción de potestas [Befugnis]. Sobre el lenguaje y origen de los derechos subjetivos, véase TIERNEY, B. The Idea of Natural Rights: Studies on Natural Rights, Natural Law and Church Law 1150-1625. Atlanta-Georgia: Scholars Press, 1997, especialmente, pp. 43-77. 
de usar ciertos objetos de nuestro arbitrio, porque nos hemos posesionado de ellos con antelación. ${ }^{28}$

A la luz de la clasificación de las acciones establecida en la "Introducción", la denominación del postulado de la razón práctica como una ley permisiva cobra un primer sentido. El postulado señalaba la posibilidad moral de hacer uso de los objetos, dado que una prohibición supondría una contradicción de la libertad consigo misma. En consecuencia, la acción vinculada con la posesión jurídica de un objeto, según la mencionada clasificación, no es prohibida ni obligatoria, sino una acción permitida. Sin embargo, no se trata de una acción permitida "moralmente indiferente" o adiaphoron, pues la acción de poseer un objeto tiene la consecuencia jurídica inmediata de imponer una obligación a los demás que antes no tenían: la de abstenerse de usar un objeto. La posesión jurídica, de acuerdo con Kant, "como relación de la persona con objetos que carecen de obligación, no es sino la relación de una persona con personas, que consiste en obligar a todas ellas, en lo que se refiere al uso de las cosas, por la voluntad de la primera". ${ }^{29}$ De allí que Kant presente el postulado como una ley permisiva: en referencia a la posesión jurídica encuentra la clase de acciones permitidas que no son moralmente indiferentes — pues implican la pretensión de imponer una obligación a los demás-, y por lo tanto, tal como lo había anunciado en la "Introducción", esa clase de acciones requiere de un permiso.

Ahora bien, por otro lado, Kant señala en el pasaje citado que la potestad conferida por la ley permisiva para imponer una obligación no se puede "extraer de los meros conceptos del derecho en general". Ello se explica por dos razones. En primer lugar, la posibilidad de hacer uso de los objetos se establece mediante el postulado, que supone una "ampliación de la legislación de la razón práctica"30 en relación al axioma del derecho, pues este último solo refiere a la relación entre las personas y no entre las personas en relación a los objetos. En segundo lugar, desde el punto de vista del principio del derecho, podríamos decir (extremando el argumento) que el acto de poseer un objeto sería ilegítimo porque implicaría la pretensión de obligar a los demás por

28. RL, p. 247 , traducción modificada.

29. $R L$, p. 268.

30. RL, p. 247. 
medio de una voluntad unilateral, y una coacción unilateral es siempre contraria al derecho. Pero esa posesión, aún cuando pudiera presentar un carácter ilegítimo desde el punto de vista normativo del axioma del derecho, debe ser, prima facie, permitida. Si Kant no emplease la idea de ley permisiva se llegaría a consecuencias indeseables y contradictorias: si se admitiese el postulado de la razón práctica y no la ley permisiva, el postulado, al establecer la posibilidad de acciones que desde el punto de vista del principio del derecho son ilegítimas (porque pretenden establecer obligaciones de manera unilateral), sería un principio jurídico que contradice al axioma del derecho. Por otro lado, si no se admitiese ni el postulado ni la ley permisiva, se anularía la posibilidad de la posesión de objetos externos al arbitrio, lo cual supone, como ya sabemos, una contradicción de la libertad externa consigo misma.

Así, la función de la lex permissiva consiste en permitir una acción que de otro modo, según el concepto y el axioma del derecho, estaría prohibida. El postulado jurídico de la razón práctica se fundamenta en la libertad: la posesión de objetos externos al arbitrio debe ser posible según un principio racional a priori, cuya validez es, por tanto, independiente de si efectivamente se instituye un estado jurídico. Sin embargo, como la posesión jurídica afecta la libertad de los demás (esto es, no es una acción moralmente indiferente), en términos del axioma normativo del derecho, requiere de un permiso. En este sentido, la identificación del postulado de la razón práctica con el concepto de lex permissiva enfatiza el carácter eminentemente intersubjetivo del derecho y la idea de que las acciones de los hombres, aquí en particular las relativas a la posesión de objetos, tienen consecuencias jurídicas sobre los demás. En efecto, el punto de partida de la doctrina de la posesión es la posesión jurídica bajo la comprensión del derecho a partir de la coexistencia e interacción de los hombres: Kant define la posesión de modo tal que la idea misma de poseer algo solo tiene sentido cuando es posible una lesión por parte de los otros, con lo cual la posesión jurídica es indisociable del hecho de que los hombres coexistan y sus acciones se afecten recíprocamente. ${ }^{31}$ Cuando se toma en consideración

31. Desde la definición misma de posesión como "aquello con lo que estoy tan ligado, que cualquier uso que otro pudiera hacer de ello sin mi consentimiento, me lesionaría" ( $R L$, p. 245) solo tiene sentido que alguien afirme que posee un objeto (y pretenda un derecho al uso privado de ese objeto) en virtud de 
la estructura argumentativa de la sección "El derecho privado", cuyo tema central consiste en la justificación de los derechos adquiridos ${ }^{32}$, se advierte que la necesidad de postular una ley permisiva junto al postulado de la razón práctica muestra que el problema fundamental en torno a la propiedad será cómo justificar a los demás que la obligación impuesta a ellos mediante la posesión de un objeto es legítima.

El punto central de la doctrina kantiana de la posesión es que la potestad conferida por la ley permisiva no constituye una autorización definitiva para imponer una obligación a los demás de manera legítima. En este punto, la hipótesis de lectura que propongo se encuentra en las antípodas de la interpretación de Byrd y Hruschka. Estos autores afirman que este concepto debe ser entendido en esta última obra como una "norma que confiere poderes" dado que confiere "una facultad moral o capacidad para ser propietario de objetos físicos." ${ }^{33}$ De acuerdo con Byrd y Hruschka, "para Kant, no es el Estado en primera instancia quien establece derechos a los objetos externos de nuestro arbitrio, porque como Kant nota, la propiedad debe existir antes de que uno entre a la sociedad civil. En cambio, para Kant la razón práctica introduce o autoriza la propiedad y el poder parental a través de sus leyes de la razón. Ello es exactamente lo que para Kant hace la ley

la coexistencia inevitable con otras personas que pueden hacer un uso de él y, con ello, lesionarlo. Para Kant, el concepto de derecho refiere a una relación normativa entre personas y por ello no tendría sentido alguno suponer que alguien solo en una isla tendría derecho a los objetos o a sus tierras a partir de un acto empírico de posesión (sea caracterizado mediante la ocupación o el trabajo individual).

32. Aquí es preciso aclarar que la doctrina de la adquisición incluye el derecho real, el derecho personal, y el derecho real-personal, así como la doctrina de la posesión jurídica supone el tratamiento de esos tres posibles objetos exteriores (una cosa, el arbitrio de otro, el estado de otro en relación conmigo) $(R L$, p. 248). En ambas doctrinas, solo el primer caso (el derecho real en la doctrina de la adquisición y la posesión de cosas en la doctrina de la posesión) corresponde a la propiedad privada. De todos modos, Kant le otorga un lugar preponderante al tratamiento de los derechos adquiridos en relación al uso de objetos (y de la tierra). Como señala Brandt, la propiedad domina la argumentación y absorbe el tratamiento de las otras clases de adquisición (BRANDT, R. Persona y cosa. Hobbes ius omnia et omnes y la teoría kantiana de la posesión del arbitrio de otra persona en el contrato, p. 189).

33. BYRD, S./ HRUSCHKA, J. Kant's Doctrine of Right, p. 106. 
permisiva (lex permissiva) de la razón práctica en la Doctrina del derecho". ${ }^{34}$ Considero que esta hipótesis de lectura no es plausible en cuanto se considera la metodología que Kant estipula para una doctrina del derecho: un principio normativo, capaz de justificar deberes jurídicos de manera legítima, tiene que cumplir tres requisitos: la universalidad, la necesidad y la reciprocidad. Frente a este requisito metodológico, la ley permisiva solo satisface una de las tres características mencionadas, la universalidad (pues, en efecto, es una ley). Examinemos qué ocurre con las otras dos características, la necesidad y la reciprocidad.

En cuanto a la necesidad, este problema ya había sido advertido por Kant en Zum ewigen Frieden, donde señalaba que el carácter contradictorio de la ley permisiva se debía a que "implicarían la obligatoriedad de una acción a la que uno no puede ser obligado", ${ }^{35}$ en la medida que los permisos estarían fundados en una "contingencia práctica" y no en una necesidad de la razón. La idea de una obligación enunciada por una ley permisiva es en sí misma contradictoria pues, para Kant, la "obligación es la necesidad de una acción libre bajo un imperativo categórico de la razón", ${ }^{36}$ y como una obligación implica una "necesidad práctica" y una "coacción", el imperativo categórico "es o bien una ley preceptiva o bien una ley prohibitiva". ${ }^{37}$

En cuanto a la reciprocidad, Kant considera que ésta es un elemento analítico del derecho: por definición, el concepto del derecho no solo es coacción, sino coacción recíproca. En efecto, en la "Introducción a la Doctrina del derecho", Kant define el concepto de derecho estricto del siguiente modo:

El derecho no puede pensarse como compuesto de dos elementos, es decir, de la obligación según una ley y de la potestad de aquel que obliga a los otros a través de su arbitrio para coaccionarlos a ello, sino que podemos establecer inmediatamente el concepto de derecho sobre la posibilidad de conectar la coacción recíproca universal con la libertad de cada uno. ${ }^{38}$

34. Idem, p. 101.

35. ZeF, p. 247.

36. RL, p. 222.

37. RL, p. 223.

38. $R L$, p. 232 , traducción modificada. 
Según este pasaje, la relación por la cual uno obliga a los demás a cumplir una obligación enunciada por una ley mediante una potestad de coaccionar (lo que es, precisamente, el modo de obligar que describe la ley permisiva) no constituye en sentido estricto una relación jurídica prima facie. La coacción, en ese caso, podría dañar la libertad de los otros, con lo cual una coacción unilateral no sería una coacción "moral" sino que implicaría una relación de fuerza. Por ello, para Kant, el derecho es por definición coacción recíproca: el único modo de que coacción y libertad universal (de todos) puedan coexistir de modo de excluir la posibilidad de que aquella constituya una lesión de ésta, es la idea de una obligación igual y recíproca para todos. En efecto, Kant define a la igualdad innata, que es un elemento analítico del derecho innato, como "no ser obligado por otros sino a aquello a lo que recíprocamente podemos obligarles" ${ }^{\prime 3}$ En este marco, la ley permisiva no puede imponer una obligación de modo legítimo y definitivo, porque, dado que esa pretendida obligación procedería de un acto unilateral y dado que esa unilateralidad no garantiza la reciprocidad de la obligación, una obligación basada exclusivamente en la lex permissiva violaría el derecho innato a la libertad.

Con todo, aún cuando la ley permisiva no autorice a imponer obligaciones a los demás de modo definitivo, la pretensión de hacerlo constituye un reclamo legítimo. La potestad que ella confiere tiene una validez subjetiva que constituye un título racional para que se establezca su validez objetiva. Kant señala que en "esta pretensión [de obligar a los demás] radica el reconocimiento de estar obligado recíprocamente con cualquier otro a una abstención pareja, en lo que respecta a lo suyo exterior ${ }^{\prime \prime} .^{40}$ Pero esta pretensión de imponer a los demás una obligación (una voluntad unilateral) generada inevitablemente en la posesión jurídica de objetos es incompatible con el criterio de universalidad, necesidad y reciprocidad que requiere un principio normativo capaz de fundar obligaciones jurídicas. Por ello, se hace necesario fundar "lo mío y tuyo exterior" en la idea de una voluntad general legisladora que garantice la reciprocidad y la igualdad de la obligación prescripta por el derecho innato. En el parágrafo $§ 8$, al finalizar el desarrollo de la doctrina de la posesión jurídica, afirma Kant:

39. RL, p. 237.

40. RL, p. 255. 
Ahora bien, la voluntad unilateral con respecto a una posesión exterior, por tanto, contingente, no puede servir de ley coactiva para todos, porque esto perjudicaría la libertad según leyes universales. Así pues, solo una voluntad que obliga a cada cual, por tanto colectivo-universal (común) y poderosa, puede ofrecer a cada uno aquella seguridad. Pero el estado sometido a una legislación exterior universal (es decir, pública), acompañada de poder, es el estado civil. Así pues, sólo en el estado civil puede darse un mío y tuyo exterior. ${ }^{41}$

En conclusión, la posesión permitida por la ley permisiva tiene una validez subjetiva, como un título racional para que luego, mediante la legislación de la voluntad general y un poder coactivo legítimo, o sea en el Estado, se establezca su validez objetiva. Considero que con esto, Kant muestra ya en el análisis de "la condición subjetiva de uso de objetos", que tiene lugar en la doctrina de la posesión, que la idea de una voluntad general legisladora oficia como único fundamento jurídico del ejercicio efectivo de los derechos adquiridos. Si la hipótesis de lectura que intenté defender a lo largo de este trabajo tiene algún asidero, tenemos entonces elementos suficientes para rechazar toda lectura de la doctrina kantiana de la propiedad que le otorgue exclusivamente al postulado jurídico de la razón práctica, formulado como una ley permisiva, la función de justificar derechos y deberes jurídicos relativos a la adquisición privada y cuya comprensión del Estado en Kant se limite a la protección y garantía de la propiedad adquirida según principios "iusnaturales" o "jurídicos-racionales" del derecho privado.

\section{Referências bibliográficas:}

KANT, I. Gesammelte Schriften. Ediciones: tomos 1-22, Berlín: Preussische Akademie der Wissenschaften; tomo 23: Berlín: Deutsche Akademie der Wissenschaften; desde tomo 24: Göttingen, Berlin, Akademie der Wissenschaften, 1900 y años subsiguientes.

MS: (1797) Die Metaphysik der Sitten, tomo VI, pp. 205-493.

RL: Erster Teil. Metapbysische Anfangsgriunde der Rechtslebre, tomo VI, pp. 205-378.

TL: Zweiter Teil. Metapbysische Anfangsgründe der Tugendlebre, tomo VI, pp. 379-491.

41. RL, p. 256 . 
ZeF: (1795) Zum ewigen Frieden, tomo VIII, pp. 341-386.

Vor. ZeF: (ca. 1794-1795) Vorarbeiten Zum ewigen Frieden, tomo XXII, pp. 155-175.

Vig.: (1793-1794) Metaphysik der Sitten Vigilantius, tomo XXVII, parte 4, pp. 479-732.

Vor. PR: (1797-1798) Vorarbeiten zum Privatrecht, tomo XXIII, pp. 279-336.

BRANDT, R. Das Erlaubnisgesetz oder: Vernunft und Geschichte in Kants Rechtslehre. In: _—. Rechtsphilosopbie der Aufklärung: Symposium Wolfenbiuttel. Berlin: Walter de Gruyter, 1982.

Persona y cosa. Hobbes ius omnia et omnes y la teoría kantiana de la posesión del arbitrio de otra persona en el contrato. In: Immanuel Kant. Política, derecho y antropología. Traducción de G. Leiva. México: Plaza y Valdéz, 2001.

BYRD, S./ HRUSCHKA, J. Kant's Doctrine of Right. A Commentary. Cambridge: Cambridge University Press, 2010.

BYRD, S. Intelligible possession of objects of choice. In: DENIS, L. (comp.). Kant's Metaphysical of Morals. Cambridge: Cambridge University Press, 2010.

GUYER, P. Kant's Deduction of the Principle of Rights. In: TIMMONS, M. (ed.). Kant's Metapbysics of Morals Interpretative Essays, Oxford: Oxford University Press, 2002.

HRUSCHKA, J. The Permissive Law of Practical Reason in Kant's 'Metaphysics of Morals'. Law and Pbilosopby, vol. 23, n 1, 2004. pp. 45-72.

KAUFMANN, M. Erlaubnis und Gesetz in der Scholastik und Kants Erlaubnisgesetz. In: ROHDEN, V. (ed.). Akten des X Internationalen Kant Kongresses. Berlin: Walter de Gruyter, 2008.

KERSTING, W. Wolhgeordnete Freibeit. Frankfurt am Main: Suhrkamp, 1993.

LUDWIG, B. Kants Rechtslebre. Hamburg: Felix Meiner, 2005.

MULHOLLAND, L. Kant's System of Rights. New York: Columbia University Press, 1990.

TIERNEY, B. The Idea of Natural Rights: Studies on Natural Rights, Natural Law and Church Law 1150-1625. Atlanta-Georgia: Scholars Press, 1997.

Kant on Property: The Problem of Permissive Law. Journal of the History of Ideas, v. 62, n. 2, 2001. pp. 301-312.

Natural Law and Property from Gratian to Kant. Journal of the History of Ideas, v. 62, n. 3, 2001. pp. 381-399. 
